\title{
Determination of 2D poloidal maps of the intrinsic $W$ density for transport studies in JET-ILW
}

\author{
Marco Sertoli, 1,2, a) Joanne Flanagan, ${ }^{2, \text { b) }}$ Mikhail Maslov, ${ }^{2, \text { c) }}$ Costanza Maggi, ${ }^{2, d)}$ Ivor Coffey, ${ }^{2,}$ e) Carine \\ Giroud, ${ }^{2, f)}$ Sheena Menmuir, ${ }^{2, g}$ ) Pedro Carvalho, ${ }^{3, h)}$ Anthony Shaw, ${ }^{2, \mathrm{i})}$ Ephrem Delabie, ${ }^{4, j}$ ) and JET \\ Contributors 5 \\ 1) Max-Planck-Institut für Plasmaphysik, Boltzmannstrasse 2, D-85748 Garching, \\ Germany \\ ${ }^{2)}$ CCFE, Culham Science Centre, Abingdon, Oxon, OX14 3DB, UK \\ ${ }^{3)}$ Instituto de Plasmas e Fuso Nuclear, Instituto Superior Tcnico, Universidade de Lisboa, Av. Rovisco Pais, \\ 1049-001 Lisboa, Portugal \\ 4) Oak Ridge National Laboratory, Oak Ridge, TN 37830, USA \\ 5) ${ }^{5}$ ee the author list of X. Litaudon et al 2017 Nucl. Fusion 57102001
}

(Dated: 2 November 2018)

The experimental method developed at ASDEX Upgrade for the determination of the intrinsic tungsten (W) density profile coupling data from the soft X-ray (SXR) diagnostic and vacuum-ultra-violet (VUV) spectroscopy has been upgraded for application to JET plasmas. The strong poloidal asymmetries in the SXR emission are modelled assuming a $\ln \left(\epsilon(\rho, R) / \epsilon\left(\rho, R_{0}\right)\right)=\lambda(\rho)\left(R^{2}-R_{0}^{2}\right)$ distribution, where $\rho$ is a flux coordinate and $R$ the major radius, $\lambda$ a fit parameter. The $\mathrm{W}$ density is calculated from the resulting $2 \mathrm{D} \mathrm{SXR}$ emissivity maps accounting for contributions from a low-Z impurity (typically beryllium) and main ion with the assumption that their contributions are poloidally symmetric. Comparing the result with the independent W concentration measurement of VUV spectroscopy, a recalibration factor for the SXR emissivity is calculated making the method robust against the decrease in sensitivity of the SXR diodes which has been observed across multiple campaigns. The final 2D W density map is checked for consistency versus the time-evolution of the $\mathrm{W}$ concentration measurement from VUV spectroscopy, toroidal rotation measurements from charge exchange recombination spectroscopy and tomographic reconstructions of bolometry data. The method has been found to be robust for $\mathrm{W}$ concentrations above a few $10^{-5}$ and in cases where the contributions from other medium-Z impurities such as $\mathrm{Ni}$ are negligible.

PACS numbers: 52.25.Fi, 52.25.Vy, 52.25.Xz, 52.50.Sw, 52.55.Fa, 52.65.Tt, 52.70.Gw, 52.70.La

Keywords: tokamaks, impurities, ICRH, transport, diagnostics

\section{INTRODUCTION}

The large mass tungsten (W) and its highly ionised states present in fusion-grade plasmas make it susceptible to centrifugal and electrostatic forces which lead to poloidal variations of its density within a flux-surface ${ }^{1-8}$. The theoretical framework describing these effects has been known for some time ${ }^{9-15}$, but only in recent years has this non-uniform poloidal distribution and its effects on the neoclassical transport of impurities been calculated selfconsistently and included in modern-day codes such as $\mathrm{NEO}^{16-18}, \mathrm{GKW}^{19,20}$. The theory has been found to be very robust against experimental results ${ }^{5-7}$, but the inclusion of the effects of ion-cyclotron-resonance heating has proven to be more challenging due to the difficulty in calculating the temperature anisotropy of the minority heated ions when the resonance layer is located on the low-field-side (LFS) ${ }^{21,22}$. Since ion-cyclotron-resonance-heating (ICRH) is planned to account for almost a third of ITER's heating power, a more thorough understanding on how it can be optimized for the control of central impurity accumulation can free electron-cyclotron-resonance-heating (ECRH) systems to be used for other purposes such as neoclassical tearing mode (NTM) control and current profile tailoring.

Diagnosing poloidal asymmetries and decoupling centrifugal effects from those induced by ICRH fast ions is very challenging. This requires either highly optimized tomographic reconstructions of pinhole cameras with many linesof-sight (LoS) and views of the plasma from different angles ${ }^{1-3,8}$ or non-standard diagnostics such as tangential

\footnotetext{
a) Electronic mail: marco.sertoli@ukaea.uk

b) Electronic mail: joanne.flanagan@ukaea.uk

c) Electronic mail: Mikhail.Maslov@ukaea.uk

d) Electronic mail: Costanza.Maggi@ukaea.uk

e)Electronic mail: Ivor.Coffey@ukaea.uk

f)Electronic mail: Carine.Giroud@ukaea.uk

g) Electronic mail: sheena.menmuir@ukaea.uk

h)Electronic mail: Pedro.Carvalho@ukaea.uk

i)Electronic mail: anthony.shaw@ukaea.uk

j) Electronic mail: ephrem.delabie@ukaea.uk
} 
pinhole cameras $^{4}$ or forward models of the impurity distribution ${ }^{7,23}$. For the calculation of the intrinsic W density at JET, a previously developed analysis methodology ${ }^{7,23}$ uses a combination of the available soft X-ray (SXR) cameras taking advantage of the different spectral sensitivities to self-consistently calculate the contributions of $\mathrm{W}$ and low-Z impurities such as beryllium (Be). This method requires the knowledge of the absolute and relative calibration of all the diode chips, and is thus subject to unknown systematic errors when the diodes' sensitivity decreases after consecutive campaigns.

The present paper describes an upgrade to the experimental method originally developed at ASDEX Upgrade (AUG) for the determination of the intrinsic tungsten density profile ${ }^{24}$ to account for poloidal asymmetries and enable its application to JET plasmas. The method does not rely on the absolute calibration of the SXR diodes and consistency checks of the results with other independent diagnostics are intrinsically part of the analysis, giving a series of confidence indicators to decide whether the results should be trusted or not. The analysis methodology is described in-depth in section II and applied to a few examples pulses in section III. Conclusions and an outlook are given in section IV.

\section{DETAILS OF THE ANALYSIS METHOD}

\section{A. Set of included diagnostics}

Since 2005 only 3 of the original 11 SXR cameras installed at $\mathrm{JET}^{25}$ have been kept in operation, drastically reducing the number of available lines-of-sight and of viewing angles into the plasma. These three cameras (figure 1) are located in three different octants and in the years have been equipped with different beryllium (Be) filters (table I). These factors have made it increasingly difficult to perform tomographic reconstructions ${ }^{26}$ and the cameras have often been used separately and for different purposes. Due to their favourable viewing geometry, $\mathrm{V}$ and $\mathrm{T}$ have been used mainly to characterizer in-out poloidal asymmetries, while $\mathrm{H}$ has been mainly devoted to MHD studies because of its high acquisition rate and low noise signals.

The impurity asymmetries in JET are mainly driven by centrifugal effects and the SXR emission is often an order of magnitude higher on the LFS than on the high-field-side (HFS), peaking close to mid radius or further out. Of the

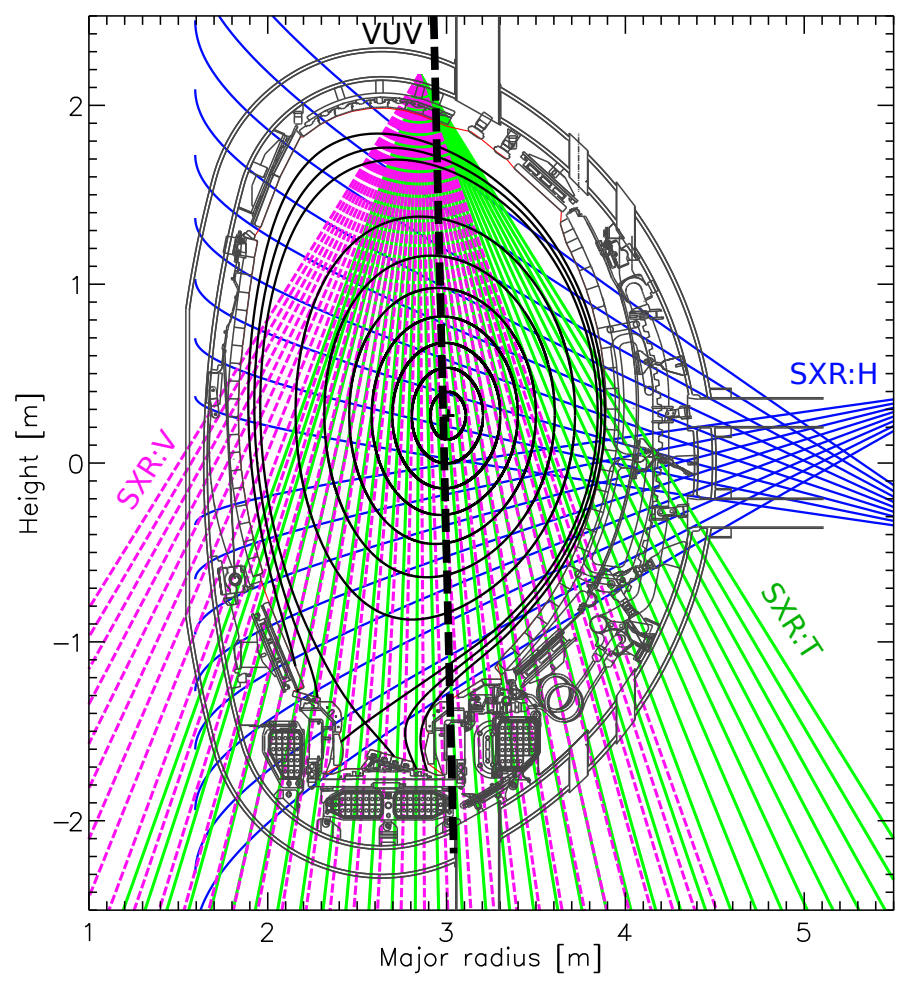

FIG. 1. Lines of sight of the SXR cameras V, T and H (magenta, green and blue respectively) and VUV spectrometer (dashed black vertical line) in JET-ILW with flux-surfaces (black) from the EFIT equilibrium reconstruction of discharge 85377 at 12 seconds in the discharge. 


\begin{tabular}{|c|c|c|c|c|c|}
\hline $\begin{array}{c}\text { Camera } \\
\text { name }\end{array}$ & Octant & Orientation & $\begin{array}{c}\text { Be-filter } \\
\text { thickness }(\mu \mathrm{m})\end{array}$ & $\begin{array}{c}\# \\
\text { of LoS }\end{array}$ & $\begin{array}{c}\text { Max acquisition } \\
\text { freq. }(\mathrm{M} \mathrm{Hz})\end{array}$ \\
\hline \hline $\mathrm{T}$ & 2 & vertical & 100 & 35 & 0.2 \\
\hline $\mathrm{V}$ & 7 & vertical & 250 & 35 & 0.2 \\
\hline $\mathrm{H}$ & 4 & horizontal & 350 & 16 & 1 \\
\hline
\end{tabular}

TABLE I. Status of the SXR diagnostic from 2015-2017: toroidal position (octant number), Be-filter thickness, number of available LoS and maximum acquisition frequency.

two cameras that can efficiently diagnose these asymmetries, $\mathrm{T}$ is positioned so to view mainly the LFS. Camera V is instead rotated of $180^{\circ}$ and the maximum impact parameter of its LoS on the LFS is approximately mid radius. This is a large limitation for the system since, for high power discharges, the peak in the SXR emissivity on the LFS is often further out. On the other hand, the calculation of the asymmetry requires the knowledge of the profile shape on the HFS as well and cannot be simply extrapolated if the information is missing. Last but not least, a faulty diode chip was installed on camera $\mathrm{T}$ in 2015, with a few channels showing strong leakage currents whose negative effects propagate to the neighbouring channels. For these reasons, the method explained here is presently applied to camera $\mathrm{V}$ only and is limited to normalized minor radii $r / a<0.6-0.7$ (where $a$ is the plasma minor radius) for the complete $2 \mathrm{D}$ profile reconstruction.

\section{B. Details of the method}

As first step, the local SXR emissivity profile is calculated in two dimensions $(\rho, R)$ using the formula:

$$
\epsilon_{\text {exp }}^{S X R}(\rho, R ; t)=\epsilon_{\text {exp }}^{S X R}\left(\rho, R_{0} ; t\right) \cdot e^{\lambda_{S X R}(\rho ; t)\left(R(\rho, \vartheta ; t)^{2}-R_{0}(\rho ; t)^{2}\right)}
$$

where $\rho$ is a flux coordinate (calculated from the equilibrium reconstruction from EFIT $^{27}$ ), $\vartheta$ the poloidal angle, $R$ the major radius of the flux-surface labelled $\rho$ at poloidal angle $\vartheta$ and $R_{0}$ the reference major radius on the HFS mid-plane (at the magnetic axis $z=z_{m a g}$ ) for the specified flux surface. Equation 1 has a major radius dependence identical to that describing the ion density asymmetries ${ }^{12}$, with the assumption that it is a good fit-function for the emissivity as well. The flux coordinate used throughout the paper is the normalized toroidal flux coordinate $\rho=\rho_{\text {tor }}=\sqrt{\left(\psi-\psi_{a}\right) /\left(\psi_{s}-\psi_{a}\right)}$ where the indices $s$ and $a$ refer to the separatrix and the magnetic axis respectively.

A first guess for the local emissivity at the reference major radius $\epsilon_{\exp }^{S X R}\left(\rho, R_{0} ; t\right)$ is calculated by performing a simple Abel-inversion of the LoSs viewing the HFS (i.e. crossing the midplane on the left of the magnetic axis) assuming a poloidally symmetric emissivity profile, while $\lambda_{S X R}(\rho ; t)$ is initially set to 0 across the whole profile. An iterative procedure then scans these two fit parameters minimizing the deviations between the re-calculated LoS-integrals and the measured values $B^{S X R}$ :

$$
\chi^{2}=\frac{1}{N} \sum_{k=1}^{N} \frac{\left(B_{k}^{S X R}(t)-\int_{k} \epsilon_{e x p}^{S X R}(\rho, R ; t) d l_{k}\right)^{2}}{\sigma_{B_{k}^{S X R}}(t)^{2}}
$$

where the sum is performed over the $N$ lines-of-sight and the integral along each LoS of length $l_{k}$. In order to avoid over-fitting, the two fit parameters are scanned over varying radial grid sizes $\delta \rho$ greater than 2 to 3 times the average difference of impact parameters of neighbouring lines-of-sight:

$$
\delta \rho_{\text {avrg }}^{\min }=\frac{1}{N-1} \sum_{k}^{N-1}\left|\rho_{k+1}^{\min }-\rho_{k}^{\min }\right|
$$

Once the best fit is found, the local emissivity is remapped to the $(R, z)$ coordinates and the W density calculated as follows:

$$
n_{W}^{S X R}=\frac{M \cdot \epsilon_{e x p}^{S X R}-n_{e}\left[n_{D} L_{D}^{S X R}+n_{B e} L_{B e}^{S X R}\right]}{n_{e} L_{W}^{S X R}}
$$

where, apart from the constant multiplication factor $M$ which will be described shortly, all other quantities are mapped to $(R, z)$ and time-dependant. The densities $n_{e}, n_{D}, n_{B e}$ of electrons, main ion (in this case deuterium) and a low-Z impurity (in this case beryllium) and SXR-filtered cooling factors $L_{W}^{S X R}, L_{D}^{S X R}, L_{B e}^{S X R}$ of the tungsten, the deuterium 
and beryllium, are all assumed to be flux-functions constant on the flux surface and remapped to $(R, z)$ according to the equilibrium reconstruction. The electron density is taken from experimental measurements, the SXR cooling factors calculated using the experimental electron temperature profiles and the atomic data from ${ }^{7,23}$. Similarly to what performed for the AUG analysis ${ }^{24,28,29}$, the $\mathrm{W}$ density is calculated only for radii where $T_{e}>1.5(k e V)$, this way accounting for the cut-off in the SXR filter-function. Moreover, in order to avoid excessive extrapolation, the radial range of the $\mathrm{W}$ density calculation is limited by the camera's LoS views and a maximum extrapolation radius is set by the average of the maximum LoS impact parameter at the LFS and HFS.

The chosen low-Z impurity for JET is typically beryllium, but, depending on the plasma scenario, seeding impurities such as neon (Ne) may also be relevant and can be used in its place. The low-Z impurity density in equation 4 is estimated using the visible Bremsstrahlung effective charge measurement $Z_{\text {eff }}{ }^{30}$ assuming it is constant across the radius and that it is governed by the chosen low-Z impurity only, i.e. $Z_{\text {eff }}(\rho ; t)=\sum_{i} n_{i}(\rho ; t)\left\langle q_{i}(\rho ; t)\right\rangle^{2} / n_{e}(\rho ; t)=$ $\left(n_{D}+n_{B e}(\rho ; t)\left\langle q_{B e}(\rho ; t)\right\rangle^{2}\right) / n_{e}(\rho ; t)$. The main ion density is then calculated from the quasi-neutrality condition $n_{D}(\rho ; t)=n_{e}(\rho ; t)-n_{B e}(\rho ; t)\left\langle q_{B e}(\rho ; t)\right\rangle$. The average charge state of the considered ion $\langle q\rangle$ (in units of the elementary charge) is calculated self-consistently with the experimental electron temperature using ADAS ${ }^{31}$ ionization and recombination rates. As already performed in the original method, all ionization balance calculations, including those for the calculation of the radiative cooling factors (SXR filtered or total), have been performed assuming local-ionization-equilibrium (LIE, also called coronal equilibrium). Transport can be neglected in the calculation of the charge state distribution in the plasma core because the ionization and recombination rates of $\mathrm{W}$ are much higher than the transport time-scales.

Once this first estimate of the W density has been calculated, a recalibration factor is evaluated by comparison with the independent W concentration measurement from VUV spectroscopy ${ }^{32,33}$ :

$$
n_{W}(R, z ; t)=\left\langle c_{W}(t) / c_{W}^{S X R}(t)\right\rangle \cdot n_{W}^{S X R}(R, z ; t)
$$

where $c_{W}$ is the VUV spectrometer measurement and $c_{W}^{S X R}$ the simulated one integrating $n_{W}^{S X R}$ from equation 4 along the line-of-sight of the VUV spectrometer and weighing it on the fractional abundance envelope of the measured ionization stages (for the details, see section IIA of ${ }^{29}$ and references therein). It is important to remember that the VUV spectrometer has been itself cross-calibrated versus bolometry data during a W ablation experiment (as is also the case for data at ASDEX Upgrade), so the quality of this calibration will affect the final results accordingly. As will be clear in section III when comparing the results to bolometry tomography, the calibration of the VUV spectrometer still seems to be quite reliable.

The re-normalization of the $\mathrm{W}$ density is the last step of the original method ${ }^{24}$ which as well does not account for low-Z impurity contributions in equation 4 . This is due to the fact that $\mathrm{W}$ typically dominates the detected SXR emission at AUG due to its high concentration $\left(c_{W} \sim 10^{-4}\right)$ as well as to the thin Be-windows $(\sim 75 \mu m)$ of the SXR diagnostic. At JET, the $\mathrm{W}$ concentration is typically an order of magnitude lower $\left(c_{W} \sim 10^{-5}\right)$ and the Be-windows are much thicker (see table I), so main ion and low-Z impurity contributions often account for more than $50 \%$ of the detected SXR emission. For this reason, another step is necessary where the re-calibrated W density (equation 5) is used to calculate the expected SXR emission, providing a final re-calibration factor for the unfolded SXR emissivity:

$$
M=\left\langle\frac{\epsilon^{S X R}}{\epsilon_{e x p}^{S X R}}\right\rangle=\left\langle\frac{n_{e}\left[n_{D} L_{D}^{S X R}+n_{B e} L_{B e}^{S X R}+n_{W} L_{W}^{S X R}\right]}{\epsilon_{e x p}^{S X R}}\right\rangle_{(R, z ; t)}
$$

where $\epsilon_{\exp }^{S X R}$ is the result of the fit routine using equation 1 and the average is performed over the whole time-range of interest and over the plasma radius within the range of validity of the analysis as defined above. Equation 4 is then re-applied using the calculated multiplication factor and the whole procedure repeated two more times.

\section{Consistency-checks versus other diagnostics}

Once the procedure has converged and the final $\mathrm{W}$ density has been calculated, a series of tests comparing the results with data from other independent diagnostics are necessary to prove the assumptions are consistent.

i) compare the time evolution of the calculated tungsten density with the independent W concentration measurement from VUV spectroscopy;

ii) evaluate the expected toroidal rotation from the observed LFS-HFS asymmetry and compare it with a rotation measurement such as that provided by charge-exchange-recombination-spectroscopy (CXRS); 
iii) calculate the total radiation using the calculated $\mathrm{W}$ density, Be (or the other chosen low-Z impurity) and main ion contributions and compare with estimates from bolometry.

These three consistency checks can validate the analysis independently from one another and, depending on their availability, can be used separately or together to strengthen the confidence in the obtained results. The comparison with VUV spectroscopy $(i)$ has been widely used in previous publications ${ }^{24,28,29}$ and does not require further explanations in this context. For the calculation of the toroidal rotation $(i i)$, equation 10 of reference ${ }^{12}$ for trace impurities in hydrogenic plasmas is used:

$$
v_{\phi}(\rho ; t)=R_{L F S}(\rho ; t) \cdot \omega_{\phi}(\rho ; t)=R_{L F S}(\rho ; t) \cdot \sqrt{\frac{2 e T_{W}(\rho ; t)}{A_{W} m_{P}} \cdot \frac{\lambda_{W}(\rho ; t)}{\left(1-\left\langle q_{W}(\rho ; t)\right\rangle \cdot \frac{A_{i}}{A_{W}} \cdot \frac{T_{e}(\rho ; t)}{T_{e}(\rho ; t)+T_{D}(\rho ; t)}\right)}}
$$

where $\omega_{\phi}(H z)$ is the toroidal rotation frequency, $T_{e}, T_{D}, T_{W}(\mathrm{eV})$ are the temperatures of electrons, main ion and tungsten respectively, $R_{L F S}(m)$ the major radius on the LFS-mid-plane where the toroidal rotation measurement from CXRS is mapped, $e(C)$ the elementary charge, $A_{D}$ and $A_{W}$ the mass numbers of the main ion and W respectively, $m_{P}(\mathrm{~kg})$ the proton mass, $\left\langle q_{W}(\rho)\right\rangle$ the average charge of $\mathrm{W}$ and the asymmetry parameter $\lambda_{W}$ :

$$
\lambda_{W}(\rho ; t)=\frac{\ln \left(n_{W}(\rho, R ; t) / n_{W}\left(\rho, R_{0} ; t\right)\right)}{R(\rho ; t)^{2}-R_{0}(\rho ; t)^{2}}
$$

In this contribution the $\mathrm{W}$ temperature is assumed equal to the main ion temperature. If ion temperature measurements from CXRS are not available, then this is set to be equal to the electron temperature.

Finally in order to compare with tomographic reconstruction of bolometry (iii), the main ion, low-Z impurity and $\mathrm{W}$ contributions are accounted for using the most up-to-date total cooling factors of the various elements $\epsilon^{\text {tot }}=$ $n_{e}\left(n_{D} L_{D}^{t o t}+n_{B e} L_{B e}^{t o t}+n_{W} L_{W}^{t o t}\right)$.

\section{APPLICATION TO EXAMPLE JET PLASMA DISCHARGES}

Three different example discharges will be analysed in order to give a comprehensive view of the wide range of cases to which the method can be applied, as well as highlight weaknesses that should be checked case-by-case. Discharges from different campaigns, performed years apart from one another have been chosen (see table II) in order to prove the robustness of the method against the decrease in sensitivity of the SXR diode chips. The effective charge $Z_{\text {eff }}$ used for the calculation and reported in table II has been estimated from the visible Bremsstrahlung measurement KS3:ZEFV; the electron density profiles are taken from either LIDAR ${ }^{34}$ or HRTS ${ }^{35,36}$ (depending on data quality); electron temperature from ECE ${ }^{37}$, LIDAR or HRTS; plasma rotation and ion temperature from CXRS. The equilibrium reconstruction is taken from EFIT without internal constraints. As already explained in the previous sections, for the present analysis only SXR camera $\mathrm{V}$ has been used. All quantities have been interpolated over a $10 \mathrm{~ms}$ resolution time axis and averaged accordingly.

\begin{tabular}{|c|c|c|c|c|c|c|c|}
\hline JPN & $\begin{array}{c}B_{t} \\
(T)\end{array}$ & $\begin{array}{c}I_{p} \\
(M A)\end{array}$ & $\begin{array}{c}P_{N B I} \\
(M W)\end{array}$ & $\begin{array}{c}P_{I C R H} \\
(M W)\end{array}$ & $Z_{\text {eff }}$ & Date & $\begin{array}{c}\text { Comments } \\
\text { on discharge }\end{array}$ \\
\hline \hline 84746 & 2.5 & 2.7 & 19 & 0 & 1.15 & August 2013 & NBI only, standard sawtooth activity \\
\hline 86614 & 2.9 & 2.5 & 23 & 4 & 1.5 & June 2014 & Hybrid discharge, highly dynamic \\
\hline 92054 & 3.3 & 2.5 & 25 & 0 & $2.5-3.0$ (increasing in time) & October 2016 & AT scenario, ion ITB, Ne-seeding \\
\hline
\end{tabular}

TABLE II. Details of the analysed JET discharges.

All three discharges are at similar plasma current $(2.5-2.7 \mathrm{MA})$, but quite different toroidal magnetic field $(2.5 \rightarrow 3.3 \mathrm{~T})$. The neutral-beam-injection $(\mathrm{NBI})$ power is $>20 \mathrm{MW}$ for all discharges (red trace in figure $2 \mathrm{a}$, axis of reference on the right), while 86614 has also $4 M W$ of ICRH used with a H-minority heating scheme at a frequency of $42 \mathrm{MHz}$ (green trace in same plot). Discharge 84746 exhibits a stable flat-top with regular, standard sawtoothing activity which is clearly visible in the cycling of the central electron temperature (red in figure 2a) and W density (red in figure 2c). The other two discharges are instead very dynamic, with $\mathrm{W}$ accumulation taking place in both 86614 and 92054. In the hybrid discharge (86614) this occurs around $9.5 \mathrm{~s}$, with the electron temperature dropping sharply but recovering before the NBI ramp-down (figure 2a). Discharge 92054 shows instead a run-away condition with strong electron density peaking (figure $2 \mathrm{~b}$ ) and a collapse of the electron temperature profile (figure $2 \mathrm{a}$ ). 

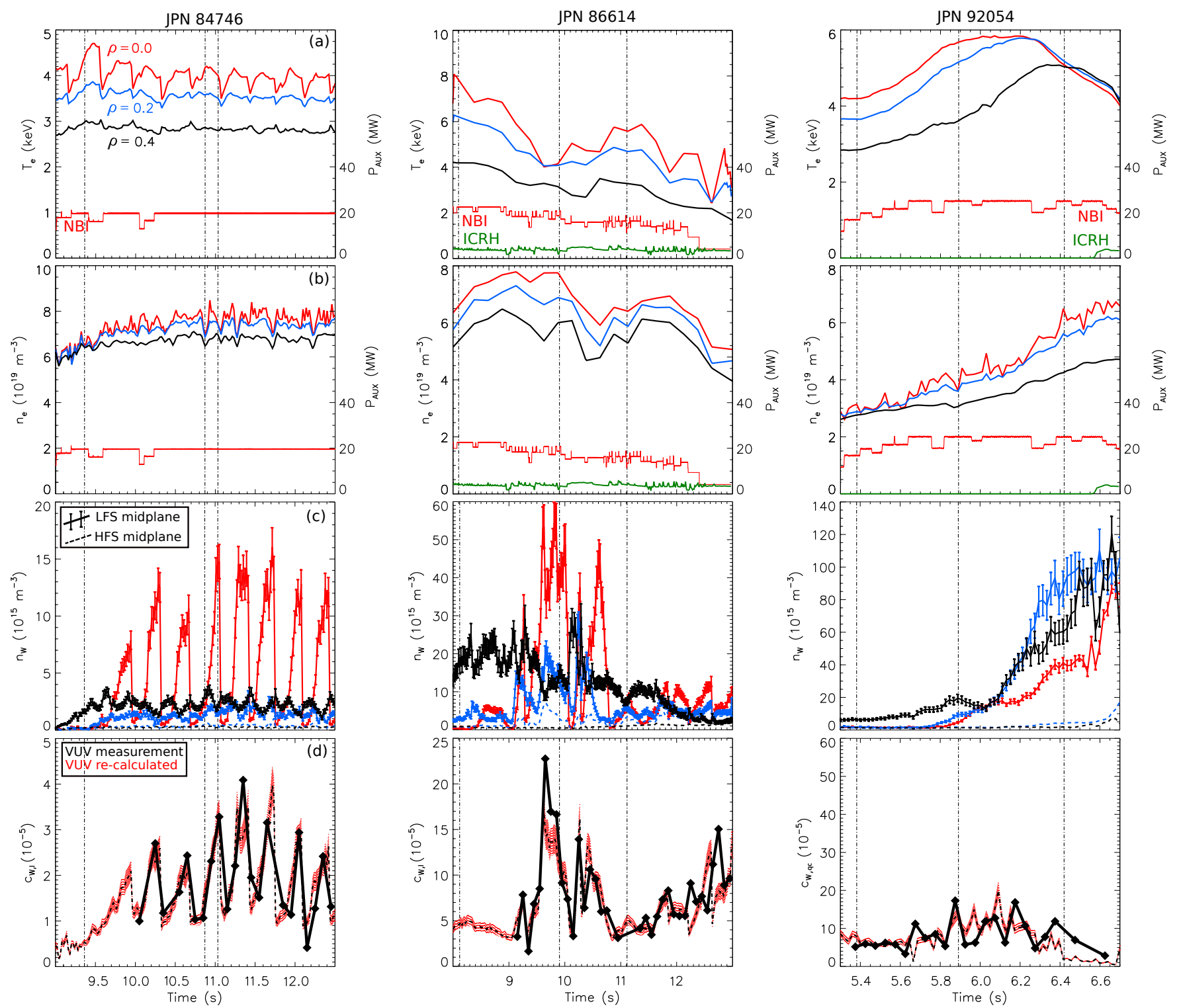

FIG. 2. Time evolution of various quantities of interest for the three discharges under analysis $(84746,86614,92309$, left to right, see plot titles): (a) electron temperature, (b) electron density and (c) W density at $\rho_{t}=0.0$ (red), 0.2 (blue) and 0.4 (black). In (d) a comparison of the VUV W concentration measurement (KT7/3, black points and solid line) and the view along its LoS of the post-calibrated SXR-evaluated W density (dashed black line with red error bars). The W density values in (c) are given on the LFS midplane (continuous lines) and on the HFS midplane at the same $\rho$ (dashed lines). In (a) and (b) the heating levels of NBI (red) and ICRH (green) are also given (reference scale on the right).

The first consistency check of the calculated $\mathrm{W}$ density is the comparison with the $\mathrm{W}$ concentration measurement using VUV spectroscopy (figure 2d). It is clear that the time evolution of the VUV measurement (black diamonds and continuous black line) is reproduced very well by the simulated value using the SXR-evaluated W density (red band, black dashed line) for all three cases and throughout the whole time range of interest. Note that for discharges 94746 and 86614 the re-normalization of the $\mathrm{W}$ density as well as the comparison of the time evolution has been performed using the VUV measurement based on the fit of W spectral lines $c_{W, l}$ (measuring around $T_{e} \sim 3.5 \mathrm{keV}$ ); for 92054 the fit on the quasi-continuum spectral feature $c_{W, q c}$ has been used (measuring around $T_{e} \sim 1.5 \mathrm{keV}$ ). The reason for this is that for discharge 92054 the spectral-lines signal is too low for a trustworthy calculation of the concentration measurement, so the more edge value had to be used (refer $\mathrm{to}^{24}$ and references therein for details on these measurements). 

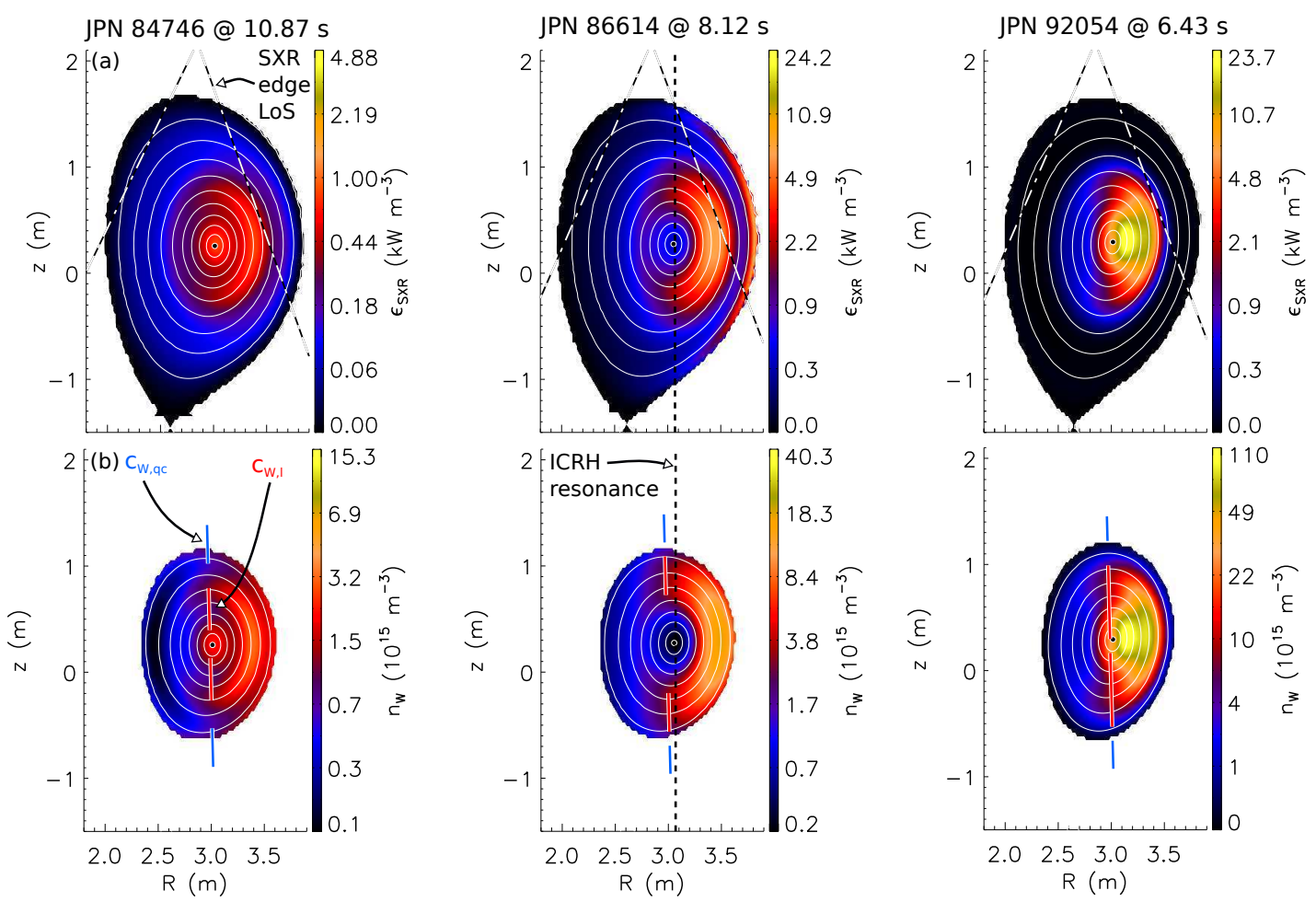

FIG. 3. 2D poloidal maps of the inverted SXR emission (a) and the final W density (b) for one time-point for each of the three discharges under analysis (see plot titles). In (a) the edge LoS if the SXR camera V are shown. In (b) the integration region for the two VUV spectrometer measurements $c_{W, l}$ and $c_{W, q c}$ (red and blue vertical lines). For 86614, the position of the ICRH resonance is also shown (dashed vertical line).

Figure 2c gives also an idea of the $\mathrm{W}$ density profile asymmetry, with the LFS midplane values at $\rho=0.2$ and 0.4 (continuous line with error bars, blue and black respectively) being up to an order of magnitude larger than those on the same flux-surface but on the HFS (dashed lines, same colour coding). This is even clearer when looking at the 2D poloidal maps of either the inverted SXR emissivity (figure 3a) or the W density (figure 3b). Similarly to what done $\mathrm{in}^{28}$, the $\mathrm{W}$ density profiles are truncated at radii where the electron temperature drops below $1.5 \mathrm{keV}$, below which the SXR instrument function is highly uncertain. Moreover, due to the limited view of the plasma of SXR camera V especially on the LFS (edge LoS shown in figures 3a), the W density is further limited to the average of the maximum impact parameter of the LoS viewing LFS and HFS $\rho<\left[\max \left(\rho_{\min , L F S}\right), \max \left(\rho_{\min , H F S}\right)\right] / 2$. Also shown in figures $3 \mathrm{~b}$ are the integration regions of the VUV spectrometer for the spectral-lines measurement $c_{W, l}$ (vertical red lines) and quasi-continuum measurement $c_{W, q c}$ (vertical blue lines). From this picture, it is clear why the re-normalization and comparison of the time-evolution is best made with the former, since the latter requires an extrapolation of the SXR-evaluated W density beyond the region of trustworthiness of the calculation.

Confidence in the inversion technique used for the evaluation of the 2D SXR emissivity can be obtained by comparing the raw SXR brightness (dashed lines and points with error bars in figure 4) with the projections of the inverted SXR profiles (continuous lines). A 10\% error is assumed on the SXR data, but, due to the large gradients in the emissivity profile close to plasma centre, in the fitting routine the weight of the central channels has been artificially modified: $2-25 \%$ increasing linearly from the centre towards the edge. The results are in general very satisfactory, as is the case for the examples considered in this publication.

Now that the confidence in the data treatment and analysis has been confirmed, the 1D profiles at different timepoints can be analysed and discussed (figure 5). The errors on the final fits of the electron temperature and density profiles (figures 5a and 5b respectively, error not shown on the plot) have been calculated propagating a $5 \%$ error assumed on the raw data and the deviation of the fit to the data. If a time-averaging of the profiles has been performed (as is the case for ECE and HRTS data on the final $10 \mathrm{~ms}$ time resolution), the standard deviation of the data points in the time window used for averaging has also been included in the propagation. The uncertainty in the $\mathrm{W}$ density (figure 5c) is then calculated propagating these errors alongside with the assumed 10\% error on the post-inversion SXR emissivity. This is clearly a lower limit, not including either the uncertainties in the atomic data used for the 

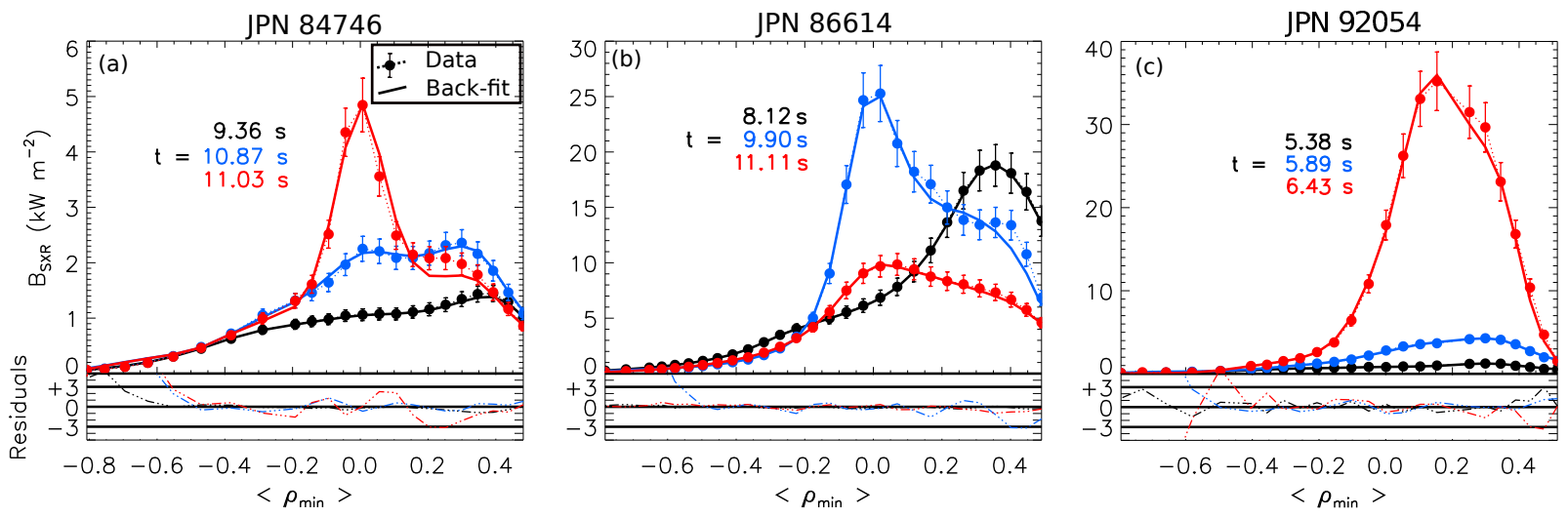

FIG. 4. Comparison of the SXR camera V brightness (points with error bars and dashed lines) with back-fitted line-of-sight integrals of the inverted emissivity for the three discharges and time-points under analysis (shown as vertical dashed lines in figures 2).

calculation of the cooling factors or possible contamination of the SXR brightness due to other medium-Z impurities. While the errors in the atomic data cannot be estimated, if the contribution of other medium-/high-Z impurities is non-negligible, this will show up in one of the consistency checks and the results rejected.

As can be seen in figure 5c, the poloidal asymmetry in the $\mathrm{W}$ density is often so large (e.g. for discharges 86614 and 92054) that the values on the LFS mid-plane (continuous lines) are up to an order of magnitude larger than those on the same flux-surface on the HFS mid-plane (dashed lines). When viewing along the LoS of the VUV spectrometer (dashed lines in figure 5d), the values match nicely not only with the more central VUV measurement (diamonds), but also with the more edge measurement using the quasi-continuum (triangles). When comparing figures 5c and 5d, as well as the 2D maps in figure 3, it is clear how much the poloidal asymmetry affects the VUV measurements, with high levels of the $\mathrm{W}$ density often missed because too far away from its vertical LoS (figure 1).

\section{Sawtooth cycling (84746)}

For discharge 84746 the time-points have been chosen with very similar electron density and temperature profiles, but very different $\mathrm{W}$ density. At the start of the discharge, the $\mathrm{W}$ density is still very hollow (black in figure 5c) with almost no $\mathrm{W}$ in the plasma centre. The sawtooth cycles then modulate this profile with the $\mathrm{W}$ density increasing of about a factor 3 inside $\rho<0.3$ while remaining relatively stable close to mid radius (blue and red). Calculating the expected toroidal rotation from the $\mathrm{W}$ poloidal asymmetries using equation 7 , the profiles match relatively well the values measured by CXRS (figure 5e), apart from deviations in the centre and at the edge of the radial range of validity of the method $(\rho \sim 0.6)$. The mismatch in the plasma centre is most probably due to uncertainties in the equilibrium reconstruction. Since it has been observed that the Shafranov shift can be overestimated by the standard EFIT reconstruction, a movement of the magnetic axis towards the HFS would increase the poloidal asymmetry and thus raise the estimated toroidal rotation close to the plasma centre. At larger radii, the difference may instead be due to profile effects of the low-Z impurity used for the calculation (presently assumed constant across the radius) or to contributions to the SXR emissivity by other impurities that are not accounted for in the method.

\section{Recovery from accumulation (86614)}

The time-points chosen for discharge 86614 describe instead a non-catastrophic event of $\mathrm{W}$ accumulation. As in the previous example, at the beginning of the time-range of interest the $\mathrm{W}$ density is hollow, with very little $\mathrm{W}$ in the plasma centre and a very strong poloidal asymmetry (black in figure 5c). The accumulation inside $\rho<0.3$ is larger than in 84746, reaching concentrations of $c_{W} \sim 5 \cdot 10^{-4}$, with the electron temperature falling of about $50 \%$ of its original value. The event is nonetheless not fatal, with the discharge recovering and proceeding smoothly up to the ramp-down of the NBI. For this case, a final consistency check is performed comparing the total radiation calculated by bolometer tomography (points in figure 5e) with the sum of all contributions used for the calculation of the $\mathrm{W}$ density (in this case $\mathrm{W}, \mathrm{Be}$ and $\mathrm{D}$, continuous lines in the plot). The match is incredibly good for the central values, with the intensity of the total radiation and width of the radiating region fitting very well the experimental value. The radiation peak on the LFS is instead underestimated quite drastically, especially for the blue case. This peak is clearly outside of the visibility region of the SXR camera (vertical black lines), so this may play a crucial role in this mismatch. On the other hand, errors in the tomographic reconstructions which are not estimated here could also play a role. 

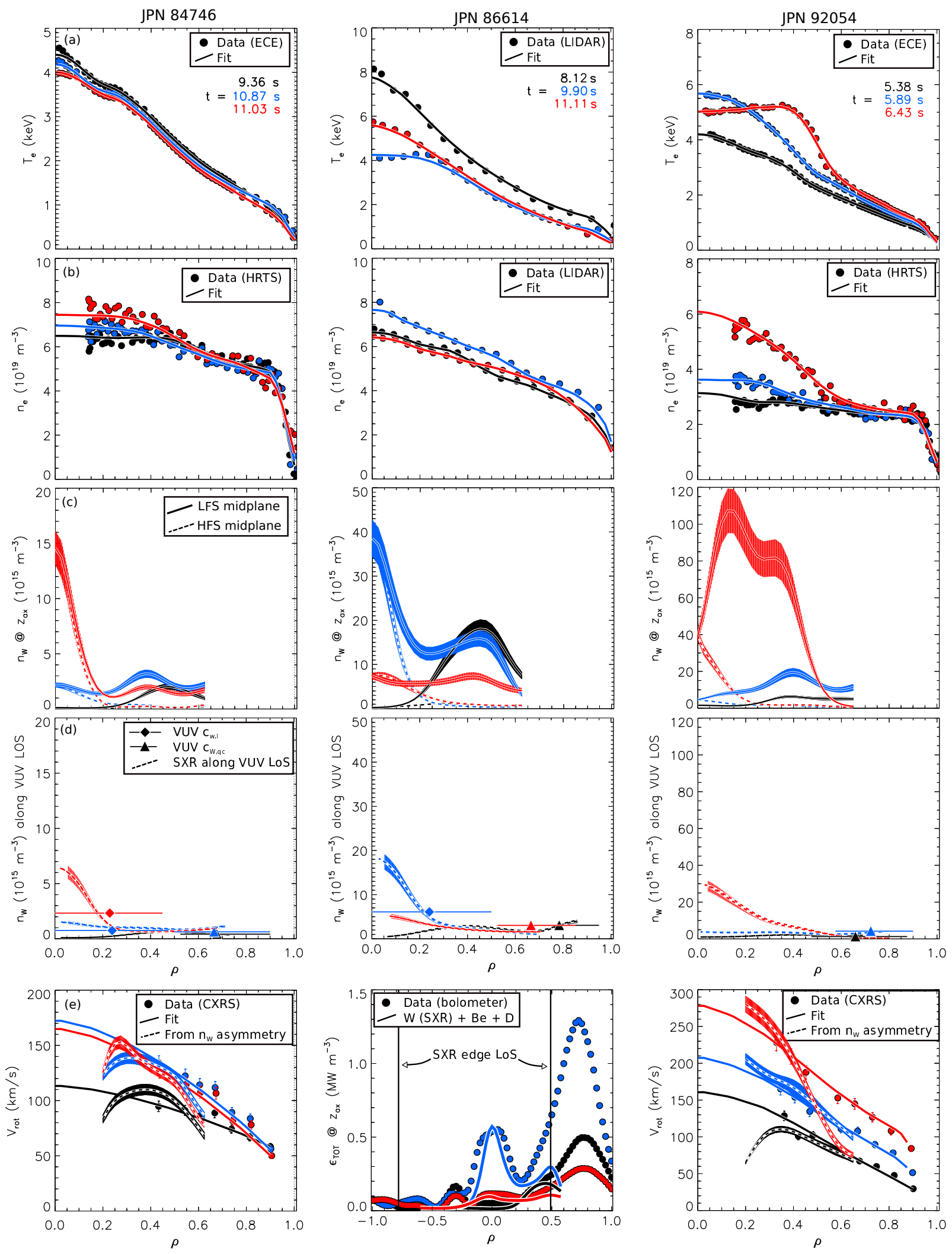

FIG. 5. Profiles of (a) the electron temperature, (b) electron density, (c) W density at midplane (LFS shown as a continuous line, HFS as a dashed line), (d) W density measured along the LoS of the VUV spectrometer (dashed lines with error bars) compared with the two measurements from the VUV spectrometer (diamonds and triangles), (e) the toroidal rotation measured by CXRS (points and continuous line) compared with the rotation estimated from the W density asymmetry (dashed lines) for discharges 84746 and 92054, the tomographic reconstruction of bolometry (points) compared with the total radiation estimated summing all the contributions used for the W density calculation (continuous lines) for discharge 92054. The time-points analysed are shown in figure 2 as dashed vertical lines. 


\section{Run-away accumulation (92054)}

Finally, the case of 92054 is very interesting because of the extreme accumulation taking place (central $c_{W} \sim 10^{-3}$ ), in the presence of strong electron density peaking (figure 5b) and a very large toroidal rotation (figure 5e). The asymmetry is such that the highest W density viewed along the VUV spectrometer line-of-sight is of the same order as the other two cases analysed, despite its maximum value is a factor $2-10$ larger (figures 5c). The increase in toroidal rotation observed by CXRS (points in figure 5e) is relatively well reproduced by the estimate from the $\mathrm{W}$ asymmetry (dashed lines). Similarly to 84746 , an underestimated Shafranov shift could account for the mismatch close to the plasma centre (black time-point). The underestimation of the asymmetry in the last time-point considered (red) is instead most probably due to a wrong treatment of low- / mid-Z impurities taking part in the SXR emission. With values of $Z_{\text {eff }}$ close to 3 at the end of the time-range of analysis and neon seeding taking place, the assumption of flat $Z_{\text {eff }}$ profile most probably does not hold.

\section{CONCLUSIONS AND OUTLOOK}

A new data analysis tool is available at JET for the estimation of the 2D poloidal distribution of the intrinsic $\mathrm{W}$ density. The method relies on the well established approach of combining measurements from the SXR diagnostic with those from a single LoS VUV spectrometer measuring solely W spectra. This has been proven to work well at JET after an upgrade necessary to account for poloidal asymmetries in the SXR emissivity. Information on the absolute calibration of the SXR diodes is not necessary, so a degradation in time of sensitivity of the diodes does not invalidate the final results. Consistency checks of the results with other independent diagnostics (e.g. VUV, CXRS and bolometry) are intrinsically part of the analysis and give a series of confidence indicators to decide whether the results should be trusted or not. The method has been tested extensively on JET-ILW data and has been found to be robust for $\mathrm{W}$ concentrations above a few $10^{-5}$ so that the SXR emissivity is not dominated by Bremsstrahlung emission, and in cases where the contributions from other medium- $\mathrm{Z}$ impurities such as $\mathrm{Ni}$ are negligible. If these contaminations are substantial, the developed consistency checks will fail thus providing a robust way to reject the results.

The calculated 2D W density will now be used for numerous physics studies in JET, e.g. to characterise the poloidal asymmetries induced by NBI and ICRH, to further understand the causes of impurity accumulation, to interpret the interactions between MHD modes and changes in impurity transport, etc. With the appropriate consistency checks, the quantities calculated by the code can also provide some important information for a more precise estimation of the total radiated power. This can then pose constraints to tomographic reconstructions of the total radiated power in the plasma core, especially in those cases where strong radiation from the inner divertor is dominating the vertical LOS of bolometry, which are also the ones usually viewing the plasma centre.

Lastly, depending on the application, care must be taken to use appropriate input data to the code in order to avoid large systematic uncertainties. For example, in order to precisely calculate the poloidal asymmetries and disentangle the effects due to ICRH from those caused by the centrifugal force, it is vital to have a precise equilibrium reconstruction with a correct estimate of the plasma centre. Calculation of transport coefficients as recently performed in $\mathrm{AUG}^{29}$ will instead require high resolution (both in time and radially) electron density and temperature profiles in order to correctly resolve the evolution of the $\mathrm{W}$ density during sawtooth cycles.

\section{ACKNOWLEDGMENTS}

This work has been carried out within the framework of the EUROfusion Consortium and has received funding from the Euratom research and training programme 2014-2018 under grant agreement No 633053. The views and opinions expressed herein do not necessarily reflect those of the European Commission.

\footnotetext{
${ }^{1}$ L. Ingesson, B. Alper, H. Chen, A. Edwards, G. Fehmers, J. Fuchs, R. Giannella, R. Gill, L. Lauro-Taroni, and M. Romanelli, Nuclear Fusion 38, 1675 (1998).

${ }^{2}$ L. C. Ingesson, H. Chen, P. Helander, and M. J. Mantsinen, Plasma Physics and Controlled Fusion 42, 161 (2000).

${ }^{3}$ H. Chen, N. C. Hawkes, L. C. Ingesson, M. von Hellermann, K.-D. Zastrow, M. G. Haines, M. Romanelli, and N. J. Peacock, Physics of Plasmas 7, 4567 (2000), https://doi.org/10.1063/1.1311806.

${ }^{4}$ M. L. Reinke, I. H. Hutchinson, J. E. Rice, N. T. Howard, A. Bader, S. Wukitch, Y. Lin, D. C. Pace, A. Hubbard, J. W. Hughes, and Y. Podpaly, Plasma Physics and Controlled Fusion 54, 045004 (2012).

${ }^{5}$ C. Angioni, P. Mantica, T. Pütterich, M. Valisa, M. Baruzzo, E. Belli, P. Belo, F. Casson, C. Challis, P. Drewelow, C. Giroud, N. Hawkes, T. Hender, J. Hobirk, T. Koskela, L. L. Taroni, C. Maggi, J. Mlynar, T. Odstrcil, M. Reinke, M. Romanelli, and J. E. Contributors, Nuclear Fusion 54, 083028 (2014).

${ }^{6}$ C. Angioni, F. J. Casson, P. Mantica, T. Pütterich, M. Valisa, E. A. Belli, R. Bilato, C. Giroud, P. Helander, and J. Contributors, Physics of Plasmas 22, 055902 (2015), http://dx.doi.org/10.1063/1.4919036.
} 
${ }^{7}$ F. J. Casson, C. Angioni, E. A. Belli, R. Bilato, P. Mantica, T. Odstrcil, T. Pütterich, M. Valisa, L. Garzotti, C. Giroud, J. Hobirk, C. F. Maggi, J. Mlynar, M. L. Reinke, and ASDEX Upgrade Team, Plasma Physics and Controlled Fusion 57, 014031 (2015).

${ }^{8}$ T. Odstrcil, T. Pütterich, C. Angioni, R. Bilato, A. Gude, M. Odstrcil, A. U. Team, and the EUROfusion MST1 team, Plasma Physics and Controlled Fusion 60, 014003 (2018).

${ }^{9} \mathrm{~F}$. L. Hinton and S. K. Wong, The Physics of Fluids 28, 3082 (1985), http://aip.scitation.org/doi/pdf/10.1063/1.865350.

${ }^{10}$ S. K. Wong, The Physics of Fluids 30, 818 (1987), http://aip.scitation.org/doi/pdf/10.1063/1.866333.

${ }^{11}$ K. Indireshkumar and W. M. S. Jr., Physics of Fluids B: Plasma Physics 5, 1850 (1993), https://doi.org/10.1063/1.860822.

${ }^{12}$ J. Wesson, Nuclear Fusion 37, 577 (1997).

${ }^{13}$ M. Romanelli and M. Ottaviani, Plasma Physics and Controlled Fusion 40, 1767 (1998).

${ }^{14}$ P. Helander, Physics of Plasmas 5, 1209 (1998), https://doi.org/10.1063/1.872629.

${ }^{15}$ T. Flp and P. Helander, Physics of Plasmas 8, 3305 (2001), https://doi.org/10.1063/1.1372179.

${ }^{16}$ E. A. Belli and J. Candy, Plasma Physics and Controlled Fusion 50, 095010 (2008).

${ }^{17}$ E. A. Belli and J. Candy, Plasma Physics and Controlled Fusion 54, 015015 (2012).

${ }^{18}$ E. A. Belli, J. Candy, and C. Angioni, Plasma Physics and Controlled Fusion 56, 124002 (2014).

${ }^{19}$ A. Peeters, Y. Camenen, F. Casson, W. Hornsby, A. Snodin, D. Strintzi, and G. Szepesi, Computer Physics Communications 180, 2650 (2009).

${ }^{20}$ F. J. Casson, A. G. Peeters, C. Angioni, Y. Camenen, W. A. Hornsby, A. P. Snodin, and G. Szepesi, Physics of Plasmas 17, 102305 (2010).

${ }^{21}$ C. Angioni and P. Helander, Plasma Physics and Controlled Fusion 56, 124001 (2014).

${ }^{22}$ R. Bilato, T. Odstrcil, F. Casson, C. Angioni, M. Brambilla, Y. Kazakov, and E. Poli, Nuclear Fusion 57, 056020 (2017).

${ }^{23}$ T. Pütterich, R. Dux, M. Beurskens, V. Bobkov, S. Brezinsek, J. Bucalossi, J. Coenen, I. Coffey, A. Czarnecka, C. Giroud, E. Joffrin, K. Lawson, M. Lehnen, E. de la Luna, J. Mailloux, S. Marsen, M.-L. Mayoral, A. Meigs, R. Neu, F. Rimini, M. Sertoli, M. Stamp, G. van Rooij, , and J. E. contributors, in Proc. of the 24th IAEA Conference Fusion Energy (CD-Rom), San Diego, USA, October 2012 (IAEA, Vienna, 2012) p. EX/P315.

${ }^{24}$ M. Sertoli, R. Dux, T. Pütterich, and ASDEX Upgrade Team, Plasma Physics and Controlled Fusion 57, 075004 (2015).

${ }^{25}$ B. Alper, S. Dillon, A. W. Edwards, R. D. Gill, R. Robins, and D. J. Wilson, Review of Scientific Instruments 68, 778 (1997), https://doi.org/10.1063/1.1147645.

${ }^{26}$ J. Mlynar, M. Tomes, M. Imrisek, B. Alper, M. OMullane, T. Odstrcil, and T. Pütterich, Fusion Engineering and Design 96-97, 869 (2015), proceedings of the 28th Symposium On Fusion Technology (SOFT-28).

${ }^{27}$ L. Lao, H. S. John, R. Stambaugh, A. Kellman, and W. Pfeiffer, Nuclear Fusion 25, 1611 (1985).

${ }^{28}$ M. Sertoli, T. Odstrcil, C. Angioni, and the ASDEX Upgrade Team, Nuclear Fusion 55, 113029 (2015).

${ }^{29}$ M. Sertoli, C. Angioni, T. Odstrcil, A. U. Team, and E. M. Team, Physics of Plasmas 24, 112503 (2017), https://doi.org/10.1063/1.4996412.

${ }^{30}$ K. Behringer, P. Carolan, B. Denne, G. Decker, W. Engelhardt, M. Forrest, R. Gill, N. Gottardi, N. Hawkes, E. Kllne, H. Krause, G. Magyar, M. Mansfield, F. Mast, P. Morgan, N. Peacock, M. Stamp, and H. Summers, Nuclear Fusion 26, 751 (1986).

31 "http://www.adas.ac.uk/,".

${ }^{32}$ J. L. Schwob, A. W. Wouters, S. Suckewer, and M. Finkenthal, Review of Scientific Instruments 58, 1601 (1987), https://doi.org/10.1063/1.1139408.

${ }^{33}$ T. Pütterich, R. Neu, R. Dux, A. D. Whiteford, M. G. O'Mullane, and ASDEX Upgrade Team, Plasma Physics and Controlled Fusion 50, 085016 (2008).

${ }^{34}$ M. Maslov, M. N. A. Beurskens, M. Kempenaars, and J. Flanagan, Journal of Instrumentation 8, C11009 (2013).

${ }^{35}$ R. Pasqualotto, P. Nielsen, C. Gowers, M. Beurskens, M. Kempenaars, T. Carlstrom, D. Johnson, and J.-E. Contributors, Review of Scientific Instruments 75, 3891 (2004), https://doi.org/10.1063/1.1787922.

${ }^{36}$ L. Frassinetti, M. N. A. Beurskens, R. Scannell, T. H. Osborne, J. Flanagan, M. Kempenaars, M. Maslov, R. Pasqualotto, M. Walsh, and J.-E. Contributors, Review of Scientific Instruments 83, 013506 (2012), https://doi.org/10.1063/1.3673467.

${ }^{37}$ E. de la Luna, J. Snchez, V. Tribaldos, J.-E. contributors, G. Conway, W. Suttrop, J. Fessey, R. Prentice, C. Gowers, and J. M. Chareau, Review of Scientific Instruments 75, 3831 (2004), https://doi.org/10.1063/1.1781376. 